\title{
The Radiologist and the Surgeon: The Journey from a Support Staff to the Most Valuable Player of the Clinical Team
}

\author{
Anand Ramamurthy ${ }^{1}$ \\ ${ }^{1}$ HPB Surgery and Liver Transplantation, Centre for Liver Disease \\ and Transplantation, Apollo Hospital, Chennai, Tamil Nadu, India
}

Address for correspondence Anand Ramamurthy, MS, DNB, PDF (Liver Transplantation), Centre for Liver Disease and Transplantation, Apollo Hospital, 21, Gream's Lane, Chennai 600006, Tamil Nadu, India (e-mail: livertransplantation.in@gmail.com; dranand_r@apollohospitals.com).

\begin{abstract}
Advancement in understanding of disease and availability of state-of-the-art tools for diagnosis and management have brought the radiologist and the surgeon closer. Close cooperation between them starting from initial presentation to postoperative follow-up spanning diagnosis, staging, treatment planning, interventions to management of disease sequelae, and complications has become the norm. This has benefited both clinicians and patients alike. Although better tools help in increasing accuracy, accuracy by itself does not imply precision. Technology cannot be a substitute for

Keywords

- communication skills

- hepatobiliary pancreatic surgery

- live donor liver transplant good clinical acumen or interaction between the surgeon and the radiologist. It is desirable that radiologists working in tertiary referral centers focus on two or three system-based specialties, so that they can keep pace with the latest developments in the field and deliver quality care. The new-age radiologist and surgeon should adapt to this changing scenario and increasing expectations. In the pursuit of clinical excellence, the radiologist has become the most valuable player in the clinical team.
\end{abstract}

Till as late as a couple of decades ago, surgical residents were taught to rely on detailed clinical history and thorough examination of the patient to diagnose pathology and plan management. Radiology played a relatively minor supporting role, and interaction between the clinician and radiologist was limited.

There was a lot of excitement when the hospital where I was pursuing my surgical residency acquired a CT scanner, among the initial few to be installed in the government

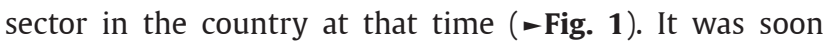
swamped by high demand making it difficult to secure appointments and compounded by breakdowns that took an inordinate amount of time to rectify. There were few radiologists trained in interpretation of the scans, and when a doubt arose, mere mortals (surgery residents) would not dare approach the head of radiology. My unit head would call the radiologist personally, and the entire team would troop to her relatively posh chamber. It was understandable why radiology residency became a popular and sought-after residency soon after.
Advancement in understanding of disease and availability of state-of-the-art tools for diagnosis and management have brought the radiologist and the surgeon closer. Close cooperation between them starting from initial presentation to postoperative follow-up spanning diagnosis, staging, treatment planning, interventions to management of disease sequelae, and complications has become the norm. This has benefited both clinicians and patients alike.

Accurate preoperative diagnosis and staging of disease has increased the proportion of surgeries being offered with a curative intent rather than diagnostic. In case of diagnostic uncertainty, image-guided biopsies are being used in difficult anatomic locations. Liver/pancreatic abscesses are managed with drugs and/or image-guided drainage, with surgery reserved for sequelae such as rupture. In unresectable liver tumors or high-risk patients, ablative procedures such as image-guided ablation (radiofrequency or microwave) are being offered with outcomes comparable to surgery. Transarterial chemo- or radioembolization
License terms

(이 (1) $\Theta \circledast$ 


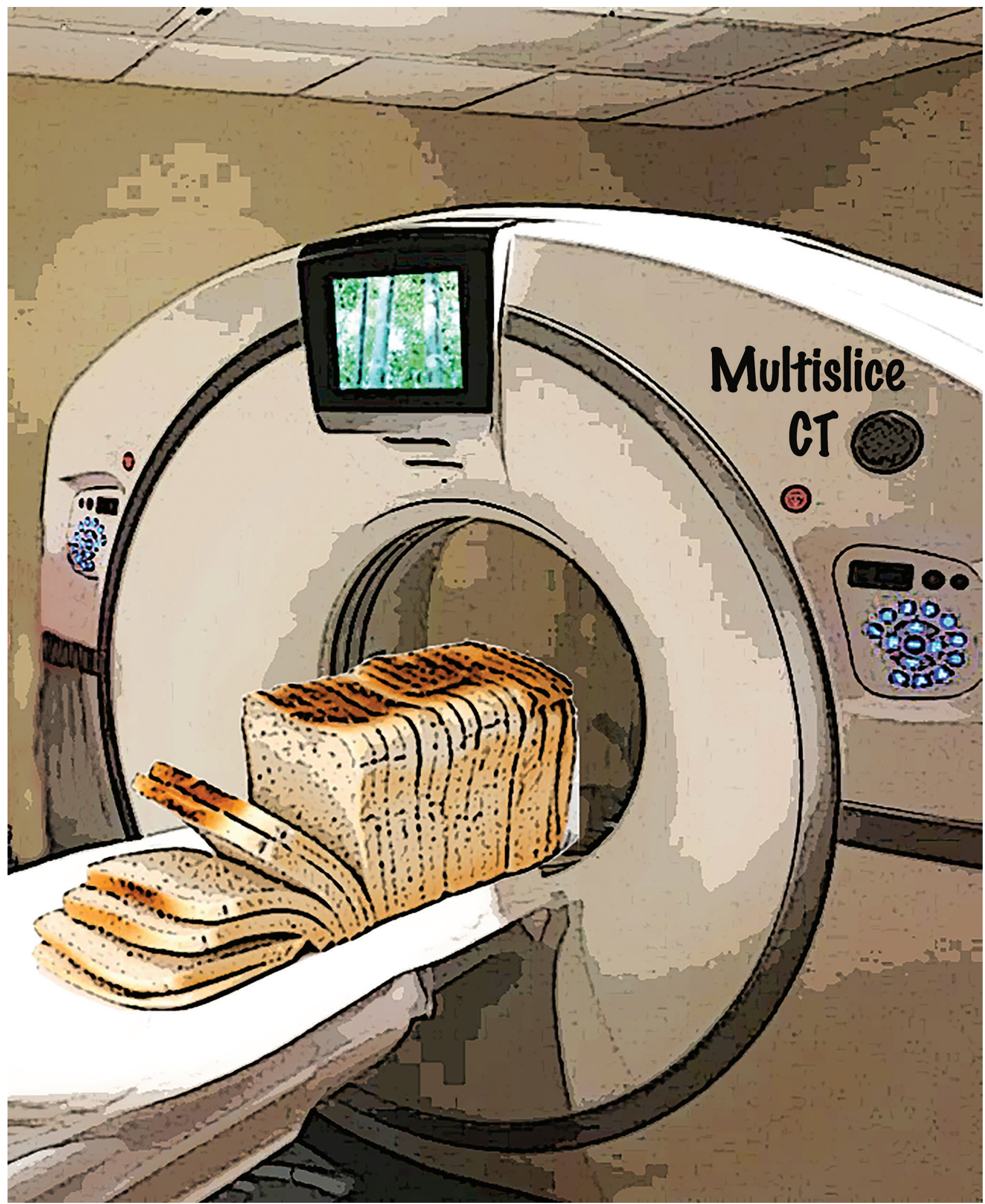

Fig. 1 Multislice CT as understood by surgeons.

has replaced therapies with low efficacy and high toxicity such as systemic chemotherapy in cirrhotic patients with hepatocellular carcinoma. It would not be an understatement to say that live donor liver transplant has been made possible and safe by imaging. Postoperative complications such as intra-abdominal collections, vascular stenosis, thrombosis, biliary leaks, or strictures are diagnosed as well as predominantly managed by the radiologist with surgery reserved for patients where intervention is either not feasible or fails to address the problem. 
There is constant evolution in the sophistication and complexity of imaging technology today. However, although better tools help in increasing accuracy, accuracy by itself does not imply precision. Technology cannot be a substitute for good clinical acumen or interaction between the surgeon and the radiologist. The brain behind the machine is more important than the machine itself. The surgeon has to provide a good clinical history with differential diagnosis, so that the radiologist knows where and what to look for and how to get the required information. The radiologist is entrusted with the responsibility of getting the best from the equipment available, confirming the diagnosis and staging the disease so that the surgeon can virtually visualize what needs to be done and how to go about it.

Maintaining ability (competence) is the first key. This implies being updated with newer developments in the field and acquisition of new skills. During the past decade when

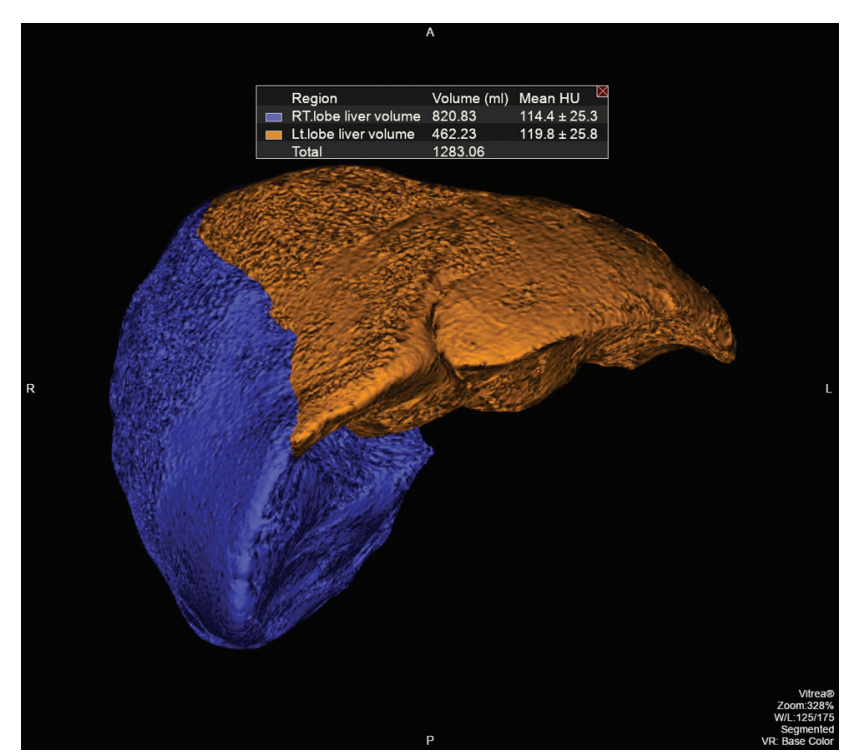

we were establishing new centers for liver transplant across southern India, we struggled with the problem of getting quality imaging across the different centers. We would wish for the understanding and expertise in imaging developed at our first program at Chennai to be transmitted across all the other centers as well. It is recommended that radiologists working in tertiary referral centers undergo focused training in two or three system-based specialties so that they can keep pace with the latest developments in the field and deliver quality care. ${ }^{1}$ Forums such as ISGAR have made it possible for radiologists to remain connected and updated as well as acquire new skills with workshops.

Affability (approachability, warmth, friendliness) is the second keyword. Both the surgeon and radiologist should be comfortable calling up their colleague to obtain the required information or walking to the radiology suite to discuss the finer details of pathology or surgical procedure. Over a period, the radiologist becomes tuned in to the requirements of the surgeon for that pathology or procedure and the required images are sent without the need for a detailed discussion or interaction. This can be aptly illustrated by our experience with live donor liver transplant surgeries. For live donor hepatectomy, details such as hepatic steatosis (liver spleen attenuation index), volumetry, and detailed vascular and biliary anatomy are required. A dedicated radiologist and technician perform imaging and post-processing of the donor films. The surgical team initially spent time with the radiologist, going over the reconstructions asking for specific views and clarifications. Today, we are provided just four films (volumetry, arterial, portal, and hepatic vein reconstruction) giving us all the information required for proceeding with a safe donor surgery (-Figs. 2-5). No more phone calls or visits are required except for complex cases. The imaging has been widely acknowledged by visitors from India and abroad as among the best transplant imaging they have seen.

Fig. 2 CT volumetry of live donor.

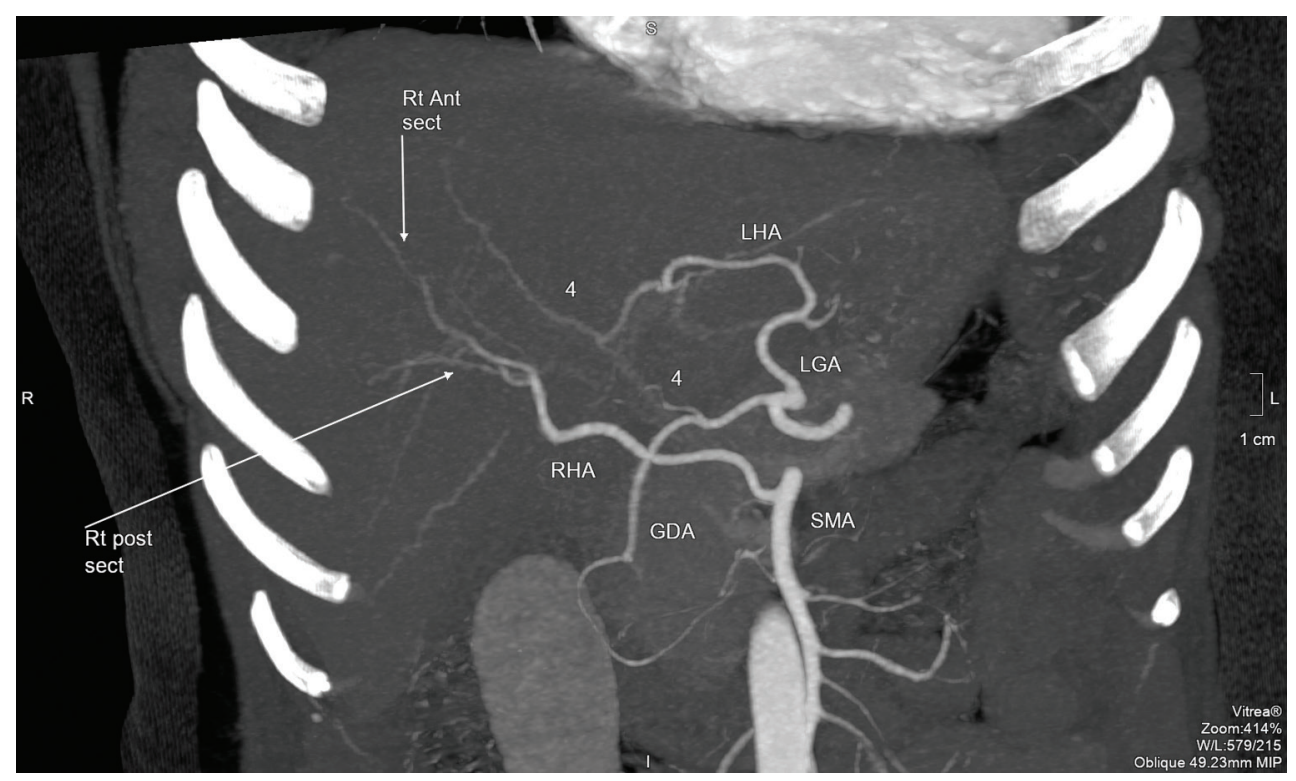

Fig. 3 Hepatic arterial anatomy. 


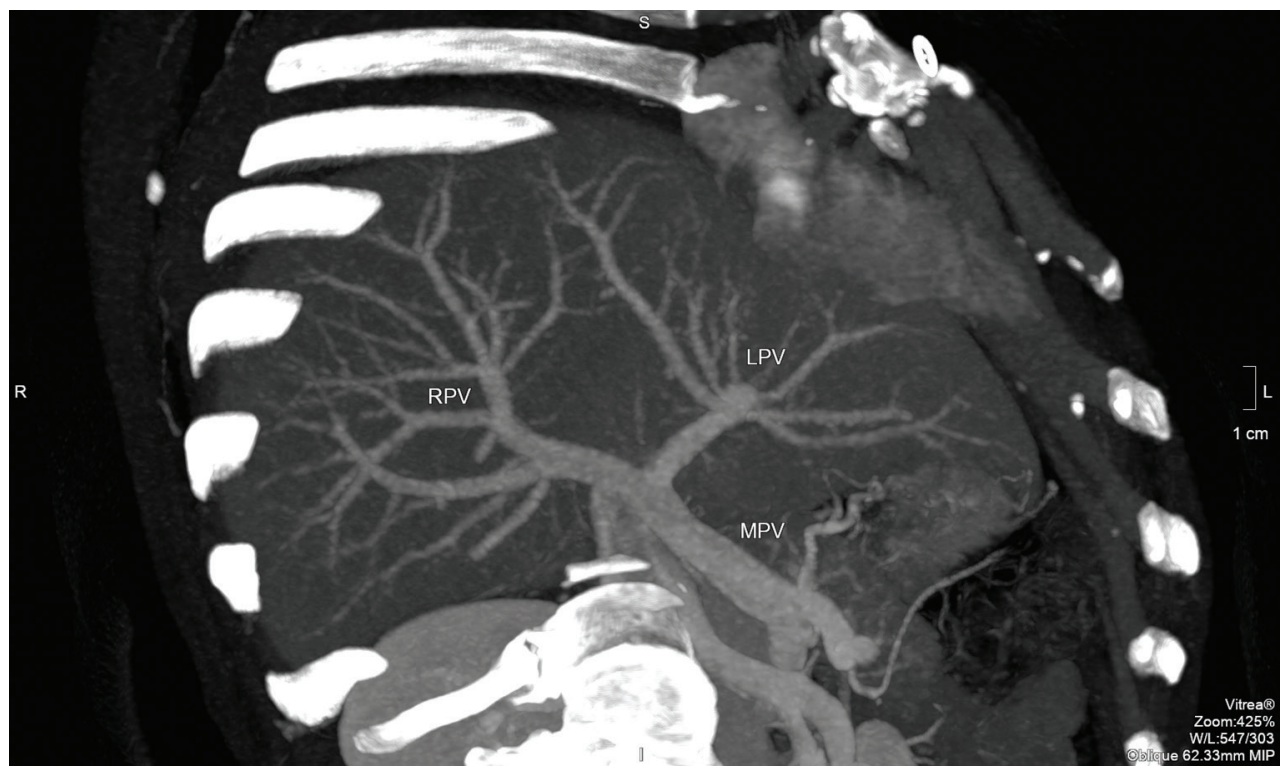

Fig. 4 Portal vein anatomy.

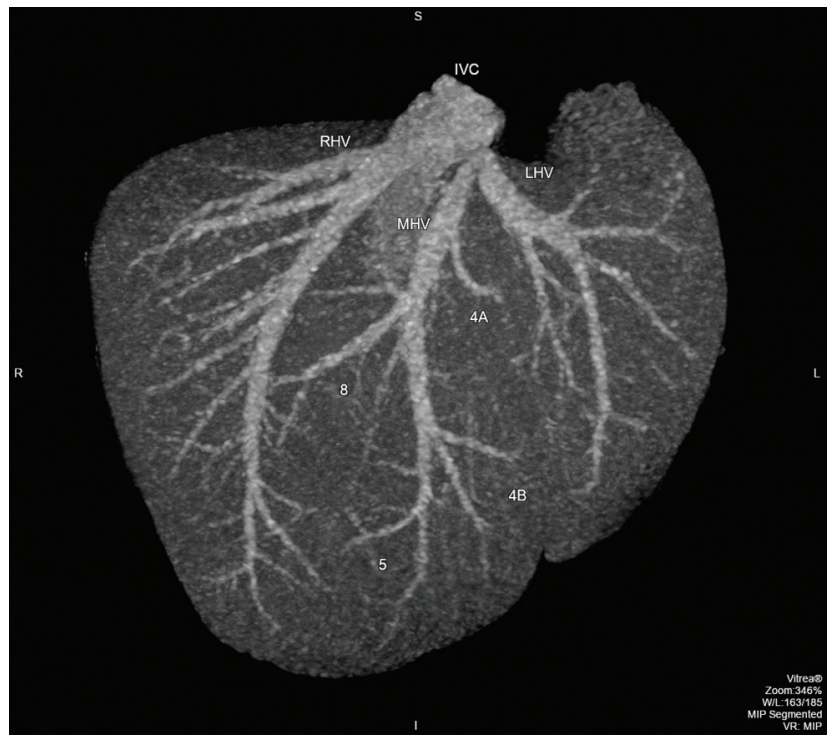

Fig. 5 Hepatic vein anatomy.

Availability is the third important requirement. Although radiology is generally considered to be an elective specialty, when called upon in the odd hours of the day or night, it is always to rescue a patient and clinician in trouble. Such a "knight in shining armor" will be remembered by the clinician as the troubleshooter and become the go-to or first recall person for elective or emergency cases in the future. Patients presenting in the emergency with trauma and major liver injury or gastrointestinal bleeding are increasingly being managed without surgery due to accurate definition of location and extent of injury by computed tomographic (CT) angiography, followed by embolization if indicated by contrast extravasation in the arterial phase.

In the era of teleradiology, reports are expected to be thorough yet clear and concise so that the referring clinician clearly understands the information. An unclear and poorly written report fails to add value to patient care. $^{2}$ Occasionally, patients visiting the outpatient clinic carry CT films with grainy images and poor contrast phasing based on which it has to be decided whether it is possible to resect the disease. The surgeon is clueless and left with no option other than to ask for a repeat imaging even though it is upsetting to both the patient and surgeon alike. The new-age radiologist and surgeon have to learn to adapt to this changing scenario and high expectations. In the pursuit of clinical excellence, the radiologist has become the most valuable player of the clinical team.

\section{Conflict of Interest}

None declared.

\section{Acknowledgment}

The author would like to thank Dr. Rochita V. Ramanan for creating the cartoon and providing the images.

\section{References}

1 European Society of Radiology 2009. The future role of radiology in healthcare. Insights Imaging 2010;1(1):2-11

2 The perfect radiology report. ACR Bulletin 2016 March; 385 